\title{
Characterization of a nitrogen-fixation (nif) gene cluster from Anabaena azollae 1a shows that closely related cyanobacteria have highly variable but structured intergenic regions
}

\author{
Donna M. Jackman and Martin E. Mulligan
}

Department of

Biochemistry, Memorial University of

Newfoundland, St John's, Newfoundland, Canada A1B 3X9

\author{
Author fivr correspondence: Martin E. Mulligan. Tel: +1 709737 7978. Fax: +1 7097372422. \\ e-mail:mulligan@kean.ucs.mun.ca
}

\begin{abstract}
The exact identity of cyanobacteria that have been cultured from symbiotic associations with the water fern Azolla spp., whether they are required in the symbiotic process, and their relationship to the symbiotic species, is a matter of some debate. We have characterized a $6 \mathrm{~kb}$ region containing the nifB operon and the nifH gene from cyanobacterium Anabaena azollae 1a, a putative symbiont of Azolla caroliniana. Five complete open reading frames have been sequenced. All are very highly conserved when compared with the corresponding regions of Anabaena sp. PCC 7120, with $93 \%$ to $97 \%$ identity at the nucleotide level and $93 \%$ to almost $100 \%$ at the amino-acid level. The intergenic regions, however, are not highly conserved (53-89\% identity) when compared to the corresponding regions of Anabaena 7120: the A. azollae genome contains both more copies and more types of short tandemly repeated repetitive sequences than Anabaena 7120. The startpoints of transcription for both the nifB and nifH operons were mapped and found to be the same as those in Anabaena 7120. It was not possible to discern an improved consensus nif promoter sequence, but it was possible to define the likely extent of the promoter to within $\mathbf{4 0}$ bases upstream of the transcription start-point.
\end{abstract}

Keywords: Anabaena azollae, cyanobacteria, intergenic region, nitrogen-fixation genes, short tandemly repetitive sequences

\section{INTRODUCTION}

The fixation of atmospheric nitrogen is restricted to select micro-organisms, among which are the heterocystforming cyanobacteria. A number of symbiotic relationships have evolved to take advantage of the nitrogenfixing ability of micro-organisms, including cyanobacteria (Evans \& Burris, 1992). One such association, which has been used in traditional agriculture, is the association between species of the water fern Azolla and heterocystforming cyanobacteria. In Azolla, cyanobacterial filaments are found inside a specialized leaf cavity. The fern stimulates differentiation of a higher-than-normal

Abbreviations: IHF, integration host factor; STRR, short tandemly repeated repetitive (sequence).

The GenBank accession numer for the sequence reported in this paper is L34879. frequency of heterocysts and the cyanobacterium provides combined nitrogen for the fern.

The cyanobacterium Anabaena azollae 1a was originally isolated from and was believed to be the endosymbiont of the water fern Azolla caroliniana (Newton \& Herman, 1979). However, there is considerable doubt as to whether this, or any other, cultured isolate is the true symbiont of Azolla spp. (Peters \& Meeks, 1989). It has not been possible to reconstitute the symbiosis between Azolla spp. cured of cyanobacteria and any cultured isolate; and restriction maps of nitrogen fixation (nif) genes in cultured isolates are not the same as those from freshly-isolated symbiotic cyanobacteria (Franche \& Cohen-Bazire, 1985, 1987; Meeks et al., 1988). The identity of the cultured isolates, whether they are required in the symbiotic process, and their relationship to the symbiotic species are of interest. 
The identification of different cyanobacterial species or strains and their relationship to one another has been difficult to resolve using traditional taxonomic morphological and physiological methods. Since nif genes are highly conserved, the nucleotide sequence of nif genes from different strains of cyanobacteria can be used as a taxonomic tool (Ben-Porath et al., 1993; Ben-Porath \& Zehr, 1994; Mathur \& Tuli, 1990). Cyanobacterial nif genes have been most extensively characterized in Anabaena sp. PCC 7120 (Anabaena 7120) (for a review see Haselkorn \& Buikema, 1992). nif genes have also been characterized from Anabaena sp. ATCC 29413 (Anabaena 29413) (Hirschberg et al., 1985; Thiel, 1993; Lyons \& Thiel, 1995), Anabaena sp. L31 (Anabaena L31) (Murphy et al., 1993), Nostoc sp. PCC 6720 (Nostoc 6720) (Beesley et al., 1994), Plectonema boryanum (Fujita et al., 1991) and Nostoc commune (Defrancesco \& Potts, 1988).

In this work, we show that related strains of cyanobacteria have distinct and different repeating sequence structure in their genomes. This observation is based on the nucleotide

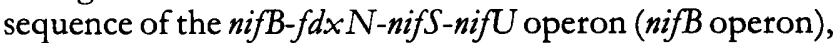
the nif $\mathrm{H}$ gene and the $5^{\prime}$ part of nifD from $A$. azollae $1 \mathrm{a}$. Our results also indicate that $A$. azollae $1 \mathrm{a}$ is very closely related to the naturally ocurring free-living strain Anabaena 29413.

\section{METHODS}

Enzymes and chemicals. All chemicals were reagent grade or better. Restriction endonucleases and other enzymes were purchased from Boehringer Mannheim, GIBCO-Bethesda Research Laboratories, New England Biolabs or Pharmacia and used in accordance with standard protocols (Ausubel et al., 1987; Sambrook et al., 1989). $\left[{ }^{35} \mathrm{~S}\right] \mathrm{dATP} \alpha \mathrm{S}\left(>1000 \mathrm{Ci} \mathrm{mmol}^{-1}\right.$; $>37 \mathrm{TBq} \mathrm{mmol}^{-1}$ ) was purchased from NEN-Dupont or Amersham. $\left[\gamma^{-33} \mathrm{P}\right]$ ATP $\left(3000 \mathrm{Ci} \mathrm{mmol}{ }^{-1} ; 111 \mathrm{TBq} \mathrm{mmol}^{-1}\right)$ was purchased from NEN-Dupont. Sequenase 2.0 was obtained from US Biochemical Corp. DNA and RNA probes for nucleic acid hybridization were prepared using digoxigenin nonradioactive labelling and detection kits (Boehringer Mannheim). Oligonucleotides used in nucleotide sequencing were the same as those used previously (Mulligan \& Haselkorn, 1989) or were purchased from the Queen's University Oligonucleotide Synthesis Laboratory (Kingston, Ontario, Canada).

Culture and preparation of nucleic acids from cyanobacteria. $A$. azollae 1a (obtained in the laboratory of R. Haselkorn, University of Chicago, IL, USA) was maintained in Chu 10 medium (Luftig \& Haselkorn, 1967) supplemented with $2.5 \mathrm{mM}$ $\mathrm{NaNO}_{3}$. Small-scale cultures $(100 \mathrm{ml})$ were passaged approximately every eight weeks by inoculating $1-5 \mathrm{ml}$ into fresh medium. Large-scale cultures of Anabaena sp. PCC 7120 (Anabaena 7120) were grown essentially as described by Belknap \& Haselkorn (1987).

DNA and RNA was prepared from large-scale cultures of cyanobacteria as described by Golden et al. (1987). DNA from small-scale stationary cultures $(100 \mathrm{ml})$ was prepared as follows. Filaments were harvested by centrifugation, washed in TEN (50 mM Tris/HCl, pH 8; $50 \mathrm{mM} \mathrm{Na}{ }_{2}$ EDTA; $0.5 \mathrm{M} \mathrm{NaCl}$ ), and resuspended in $5 \mathrm{ml}$ TEN. Strains with mucous polysaccharide sheaths were incubated for $30 \mathrm{~min}$ at $4{ }^{\circ} \mathrm{C}$ with constant mixing. Occasionally, $25 \mu \mathrm{g}$ lysozyme was added during this incubation. Equal volumes of sterile glass beads (150-200 $\mu \mathrm{m}$; Sigma) and phenol $/ \mathrm{CHCl}_{3}(1: 1, \mathrm{v} / \mathrm{v})$ were added and suspensions were

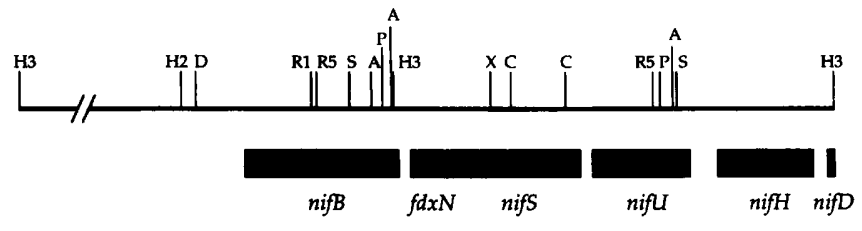

Fig. 1. Map of the $A$. azollae 1a nifB operon, nifH gene and flanking sequence. A partial restriction map of the $6061 \mathrm{bp}$ region is shown. The major restriction sites that were used in cloning and sequencing are indicated: $A, A c c l ; D, D r a l ; H 2$, Hincll; H3, HindlII; P, Sspl; R1, EcoRI; R5, EcoRV; S, Scal; X, Xbal. Only one of the Hincll sites in this region is shown. The nifB, $f d x N$, nifs, nifU and nifH genes and the $5^{\prime}$ end of nifD are indicated by the shaded boxes.

vortex mixed three times for $3 \mathrm{~min}$. Suspensions were kept on ice between vortexing. Following centrifugation at 10000 r.p.m. for $10 \mathrm{~min}$, the supernatant was removed and reextracted once with phenol and once with phenol/CHCl $3(1: 1$, $\mathrm{v} / \mathrm{v})$. Nucleic acids were then precipitated with ethanol or propanol and resuspended in TE $(10 \mathrm{mM}$ Tris $/ \mathrm{HCl}, \mathrm{pH} 8$; $0.1 \mathrm{mM} \mathrm{Na} \mathrm{N}_{2}$ EDTA). Solutions were extracted twice with phenol and once with $\mathrm{CHCl}_{3}$. Ethanol was added to precipitate the nucleic acids; where possible, DNA was spooled using a sealed Pasteur pipette and resuspended in TE.

Nucleic acid hybridizations. Southern blots of restriction digests of DNA, fractionated by agarose gel electrophoresis, were transferred to Hybond $\mathrm{N}+$ (Amersham) nylon membranes by alkaline transfer (Reed \& Mann, 1985). Recombinant clones were identified from size-directed libraries by screening colonies using Colony-Plus (Dupont) nylon membranes. RNA:RNA blots were prepared as described previously (Mulligan \& Haselkorn, 1989) using total RNA (50 $\mu \mathrm{g}$ per lane) prepared from cells grown under either nitrogen-replete $(\mathrm{N}+)$ or nitrogen-limited $(\mathrm{N}-)$ conditions. All membranes were hybridized for $40 \mathrm{~h}$ at $60^{\circ} \mathrm{C}$ with digoxigenin-labelled DNA or RNA probes.

Following hybridization, membranes were washed twice for $5 \mathrm{~min}$ at room temperature with $1 \times \mathrm{SSC}(0.15 \mathrm{M}$ sodium chloride, $0.015 \mathrm{M}$ sodium citrate, $\mathrm{pH} 7$ ) plus $0.5 \% \mathrm{SDS}$; then washed three times for $20 \mathrm{~min}$ at $60^{\circ} \mathrm{C}$ with $0.5 \times \mathrm{SSC}$ plus $0.5 \% \mathrm{SDS}$; and finally washed twice for $10 \mathrm{~min}$ at room temperature with $0.5 \times$ SSC. Bound probe was detected using an alkaline-phosphatase-conjugated anti-digoxigenin antibody followed by colorimetric detection according to the manufacturer's directions.

DNA cloning and sequencing. We isolated a $4 \cdot 1 \mathrm{~kb}$ HindIII fragment of $A$. azollae 1 a from a size-directed library of HindIII fragments cloned in the plasmid pUC19 by using a $1.8 \mathrm{~kb}$ HindIII fragment, An154.3 (Rice et al., 1982), containing the Anabaena 7120 nifH gene, as a probe. Subsequent restriction and nucleotide sequence analysis showed that the $4 \cdot 1 \mathrm{~kb}$ fragment is equivalent to both of the Anabaena 7120 clones AnH20.1 (Golden et al., 1987) and An154.3 and that it contains the $3^{\prime}$ end of nifB, the $f d x N$, nifS, nif $U$ and nifH genes as well as the $5^{\prime}$ end of nifD. An adjacent $6.4 \mathrm{~kb}$ HindIII fragment (Fig. 1) containing the remainder of the nifB gene was subsequently cloned using a $700 \mathrm{bp}$ EcoRI-HindIII fragment from Anabaena 7120 as a probe. These clones were mapped using selected restriction endonucleases to identify suitable enzymes for subcloning and sequencing (Fig. 1).

The complete nucleotide sequence of a $6061 \mathrm{bp}$ region of $A$. azollae 1 a containing the nifB operon as well as the nif $H$ gene and 
the $5^{\prime}$ part of the nifD gene was determined after subcloning appropriate fragments in $\mathrm{M} 13 \mathrm{mp} 18 / 19$ or in the plasmids pUC18, pUC19, pBR322, pSP73 (Promega), or pK184 (Jobling \& Holmes, 1990). Sequence was determined on both strands using the dideoxynucleotide sequencing method with Sequenase (US Biochemical Corp.), following the recommended protocol.

Analysis of gene expression. Primer extension analysis was performed as described previously (Mulligan \& Haselkorn, 1989) using total RNA (50 $\mu \mathrm{g}$ per reaction) prepared from cultures of $A$. azollae 1a grown under either $\mathrm{N}+$ or $\mathrm{N}-$ conditions. Either a nif $\mathrm{H}$-specific or a nifB-specific oligonucleotide (see Fig. 2) was annealed to the RNA and extended using avian myeloblastosis virus reverse transcriptase (Pharmacia).

RNA: RNA hybridization analysis was performed using either a nifH-specific or a nifB-specific digoxigenin-labelled RNA probe. Both probes were prepared by transcribing cloned fragments of the appropriate $A$. azollae 1a genes with T7 RNA polymerase. The nif $H$-specific probe was prepared from a 449 bp HincII fragment cloned in pSPT18 (Boehringer-Mannheim); the nifB-specific probe was prepared from a 436 bp Bst $\mathrm{UI}$ fragment cloned in PSPT19.

Computer analysis. Routine analysis of nucleic acid sequences was performed using the DNAlysis program of Dr Bill Buikema (University of Chicago, IL, USA). Comparisons of nucleic acid and deduced amino acid sequences were calculated using the ALIGN program of the FASTA suite of programs for the Apple Macintosh (Pearson \& Lipman, 1988). Sequence entries were retrieved from the GenBank database using the Indiana University (IUBIO) Gopher Server (Gilbert, 1993; Parker, 1993). Analysis of integration host factor binding sites was performed using the MacTargSearch program (Goodrich $e t$ al., 1990). The MulFold program was used to calculate free-energies of stem-loop formation (Jaeger et al., 1989; Zuker, 1989).

\section{RESULTS}

\section{nif gene organization}

We have characterized a $6061 \mathrm{bp}$ region of $A$. azollae 1a (Fig. 2) that contains the nifB operon as well as the nifH gene and the $5^{\prime}$ part of nifD gene in a contiguous arrangement. The $A$. azollae 1 a vegetative cell genome does not contain a $55 \mathrm{~kb}$ element interrupting the $f d x N$ gene and has a gene arrangement identical to that of the Anabaena 7120 heterocyst genome in this region. The nifH gene follows the nifB operon with a gap of $265 \mathrm{bp}$ which is similar in length to that in many other species of cyanobacteria. There are no open reading frames between the end of nif $U$ and the transcription start-point of the nif $H$ operon $-A$. azollae 1 a does not contain a cyanoglobin gene between nif $U$ and nifH as in Nostoc commune (Potts et al., 1992) and Nostoc sp. MUN 8820 (Nostoc 8820) (T. J. Belbin \& M. E. Mulligan, unpublished results).

\section{Coding region sequences}

The nucleotide and inferred amino acid sequences of the six coding regions that are reported here are all highly similar to the corresponding regions of Anabaena 7120 (Table 1). Identities range from $97.4 \%$ (nifB) to $93.4 \%$ $(f d x N)$ at the nucleotide level, and from $99.6 \%$ (NifB) to $93.1 \%(\mathrm{FdxN})$ at the amino-acid level. Table 2 shows the degree of identity between the $A$. azollae 1 a nifH sequence and that of four cyanobacteria for which the complete sequence of the nif $H$ gene has been determined. At the amino-acid level, values range from $99.7 \%$ identity for the Nostoc 6720 gene product to $84.5 \%$ identity for that of $P$. boryanum, which does not form heterocysts. Our values are consistent with those obtained in a study of partial nif $H$ sequences for four heterocyst-forming and four nonheterocyst-forming cyanobacteria using PCR amplification (Ben-Porath \& Zehr, 1994). In that study, values ranged from 94 to $98 \%$ for the heterocyst-forming cyanobacteria and from 85 to $94 \%$ for the non-heterocystforming cyanobacteria. The Anabaena 7120 gene product is longer than other $\mathrm{NifH}$ gene products, with five additional amino acids at the carboxy-terminal end, and all of the other cyanobacterial sequences contain a single amino-acid insertion (Ile 251 in the $A$. azollae 1 a sequence) that is not found in Anabaena 7120.

\section{Intergenic regions}

In general, the level of similarity between the intergenic regions of $A$. azollae $1 \mathrm{a}$ and those of Anabaena 7120 is not very high. In the interval between nifB and $f d x N, A$. azollae 1 a and Anabaena 7120 are only $52.6 \%$ identical. For the other intergenic regions the figures are: between nifS and nifU, $59 \cdot 3 \%$; between nifU and nif $\mathrm{H}, 73.9 \%$; and between nifH and nifD, $72.3 \%$. The highest overall level of identity occurs upstream of nifB, where $88.5 \%$ identity was found. Since this region contains the nifB promoter, it is not surprising that this nucleotide sequence is conserved. Most of the difference upstream of nif $B$ is due to the presence of a $40 \mathrm{bp}$ insertion in $A$. azollae 1 a that is not present in Anabaena 7120 (Fig. 2).

The intergenic regions, which are defined here as the nucleotide sequence between one coding region and the adjacent coding region and include the stop codon, contain some very interesting features. Most striking is the occurrence and diversity of short tandemly repeated repetitive (STRR) sequences. These sequences are a prominent feature in heterocyst-forming cyanobacteria (Mazel et al., 1990). They have been noted in the nifB operon of Anabaena 7120 (Mulligan \& Haselkorn, 1989) and three STRR sequences were described in Calotbrix sp. PCC 7601 (Mazel et al., 1990).

Here, we describe six new STRR sequences which we have tentatively named following the convention of Mazel et al. (1990). Fig. 3 is an alignment of the intergenic regions of $A$. azollae 1a, Anabaena 7120 and Anabaena L31 in which the STRR sequences are shown. In some cases, the STRR sequences include the stop codon of the preceding gene; hence its inclusion in our definition of an intergenic region.

A. azollae 1 a has a greater abundance and variety of STRR sequences in the nifB operon than does Anabaena 7120 . In the intergenic region between nifB and $f d x N$, the $A$. azollae 1a genome contains two sets of STRR sequences even though this interval is 40 nucleotides shorter than the corresponding interval of Anabaena 7120 . Five copies 
/Hinc2

1 AACAATTAGCCCAACAGCCCTTGAATACTTGCTGTAGTTGGGTTACAAATGAGTACAATCAGCTCCTGAACCCCTTTCCATAAATACTCGTACCCCAACTAGAAAATACTAGAACCCCTA 120 121 CCTGGAAAAAATTTTAAAATTCTCAAAACGTCCTTGAAATCATCATTTGAGATTrTGAGTATAACTGTAATAAATCCAATAAATTCTATTCCAGAGATACAAACTGTAATAGTCGAAGA 240 241 CGACTGCTATAAATCTTATATCGCCAATCACTTAACGCTTCTACATAAAACGCAAGCAAAAAGTGAGTGGAGGGATGAGATAGCACCATGCCATCTGGAATAGAAGTCGCGCGCCAGTTE 360

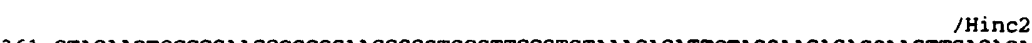
361 CTACAAGTCGGGGAACCCGCCCAACGCCCTGGCTTGGCTGTAAACACATTCTACCAAGACACCAAGTIGACACACACCAGTCCAAAACCAAGGACTTTATAGCCTGCCCTACTCAGCGAA 480 481 GCCGCTACAGGCAATGTGGCAGACGCGACTTCAATTCTTCGGGCAAACACAATCAGCAACGGCATCTCCCACAGTGGCAGCTAGTCCACCGACATTTGCGAAATTCAATGACACCACCAG 600

601 TTACAGGCTCTTCCGTTACTGAATCGACACCTACCAAAGCAAAATCAGGTGGTTGCGGATGCGACACCAGCACCACCGTGGAAATGGACGAAAAGCTCCAAGAACGTATTGCTAAACATC 720

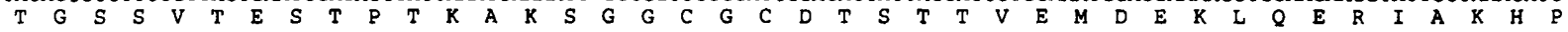
721 CCTGCTACAGCGAAGAAGCTCACCACCATTACGCGAGAATGCACGTTGCTGTTGCACCTGCTTGCAACATTCAATGCAACTACTGCAACCGAAAATATGACTGCGCTAACGAAAGCCGTC 840 841 CTGGCGTAGTTAGTGAATTACTCACACCAGAAGAAGCAGCACACAAAGTCTTAGTAATTGCAGGCAAAATTCCCCAAATGACAGTTCTGGGAATTGCTGGCCCTGGCGATCCTCTGGCGA 960

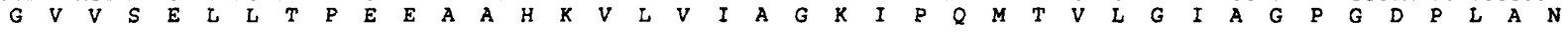
961 ACCCAGAAAAGACTTTCCGCACCTTTGAATTGATTGCAGACAAAGCCCCAGATATTAAGCTGTGTCTATCAACCAATGGTTTGATGCTGCCGGAATATGTCGATCGCATTAAACAACTAA 1080

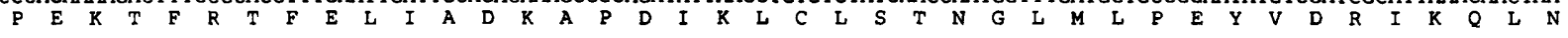
/ECORI

1081 ATATCGACCACGTTACTATTACCCTAAATACTATTGATCCAGAAATCGGCGCACAGATTTATTCTTGGGTTCACTACAAACGCAGACGTTATAGAGGTGCGGAAGGCGCGAGAATTCTCT 1200

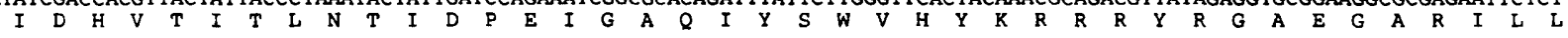

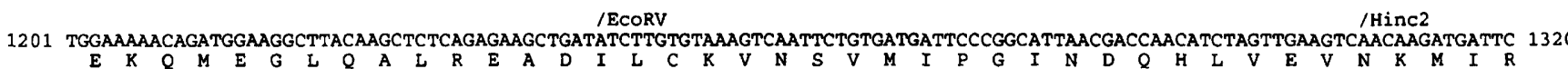

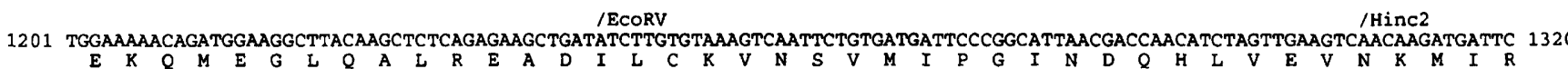

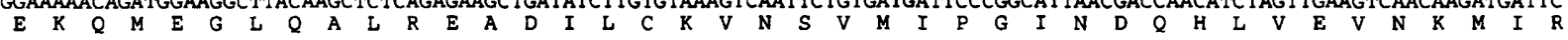

1321 GGGAACAAGGTGCATTCTTGCACAACATTATGCCTTTGATTTCTGCACCAGAACACGGTACACACTTTGGCTTAACTGGTCAACGGGGACCTTCACAAAAAGAACTGAAGTCAGTGCAAG 1440

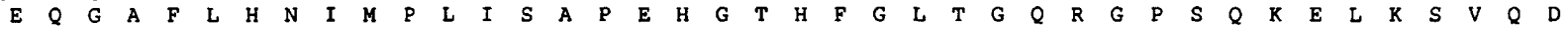

1441 ACCAATGTTCTGGCAACATGAAAATGATGCGCCATTGCCGCCAGTGTCGTGCTGATGCTGTAGGCTTGTTAGGAGAAGACCGCAGCCAGGAATTTACCAAAGATAAATTCCTCGAAATGG 1560

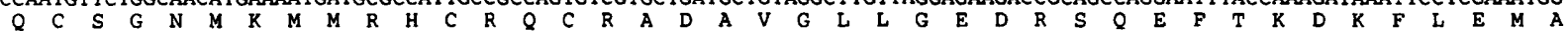

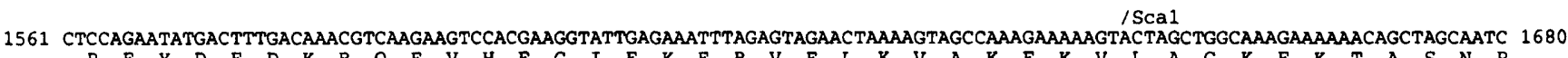

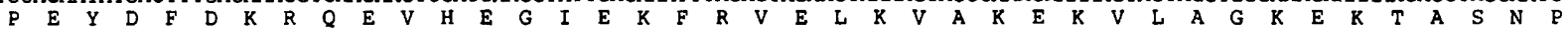
/Hinc2

1681 CTAAAATCTTAGTTGCAATAGCCACCAAAGGCGGGGATTAGTTAACCAACACTTCGGCCATGCCAAGGAATTTCAGGTTTACGAAGTAGACGGTAGTGAAGTTCGCTTCGTCAGTCACC 1800

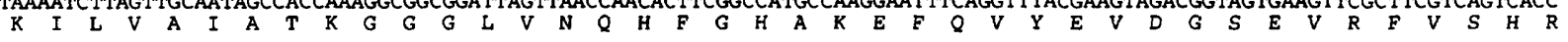
ISspl

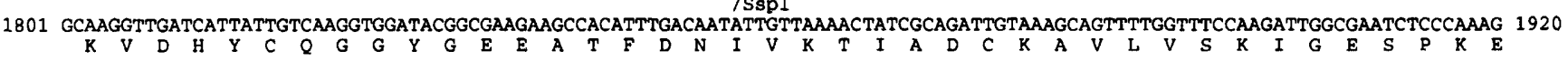
/ACC1 /Hind3

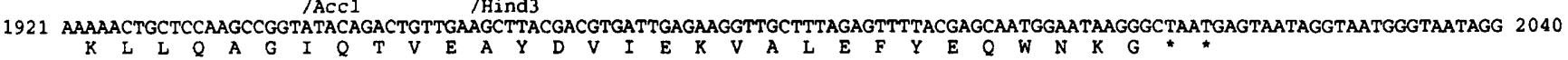

2041 TAATGGAGAGAAAACAATCATCAATTACCAATTACCAATTACCAATTACCAATCCCCACTCATCCAATCAACAAGGAGAATAATCATGGCTTACACAATTACTAGCCAATGTATTTCCTG 2160 EdxW> $M$ A

2161 CAAGCTCTGTTCGTCTGTATGCCCCACTGGTGCAATTAAGGTCGCCGAAGACGGACAGCACTGGATTGACCAAGCACTGTGTACAAATTGCGTTGATAGCGTTCACACCGTACCTCAATG 2280

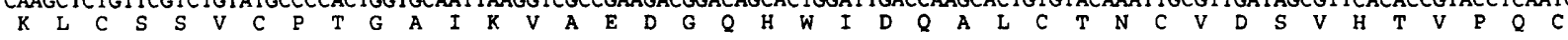

2281 TAAAGCTGGCTGTCCCACTTGCGATGGTTGCGTTAAAGTACCTAGCGATTATTGGGAAGGCTGGTTTGCTAACTACAACCGAGTTATAGCGAAATTGACAAAAAAACAAGACTATTGGGA 2400

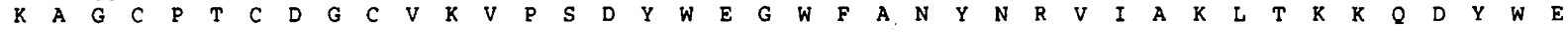

2401 ACGTTGGTTTAATTGTTATTCTCAGAAATTTTCCGAACAAATTCAAAAGCATCAGGGTGAAATCTTAGGGGATAAAGATGAGTGTTATTTATCTCGATAATAATGCTACCACTAAGGTA 2520

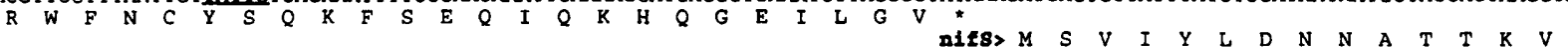

2521 GATCCAGAGGTTGTAGAGGCAATCATGCCTTACCTGACCGATTATTACGGCAATCCTTCCAGTATGCACACCTTTGGTGGACAACTGGGAAAGGAGTGAGAACAGCGAGAGAACAAGTT 2640

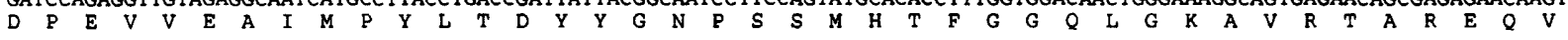

2641 GCTGCCCTGTTGGGAGCCGATGAATCAGAAATTGTTTTTACGAGTTGCGGAACCGAGGTGATAATGCTGCCATTCGTGCCGCATTGTTAGCCCAACCGGAAAAACGCCACATCATTACA 2760

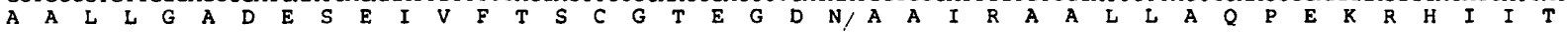

2761 ACCCAGGTTGAACACCCCGCAGTCTTAAATGTCTGCAAACAATTAGAAACCCAAGGCTACACTGTTACCTATCTTTCGGTAAATGGTCACGGGCAATTGGATCTAGATGAACTAGAAGCC 2880

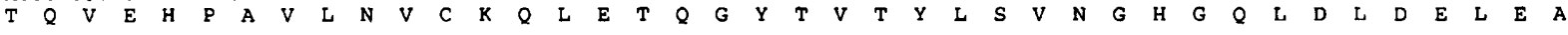

2881 TCGCTGACAGGTAACACCGCCCTGGTGACAATTATGTATGCGAACAACGAAACCGGGACTGTATTCCCAATCGAAGAGATTGGGAAACGAGTTAAGGAACGTGGCGCAATTTTCCATGTG 3000

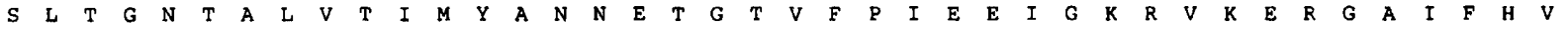

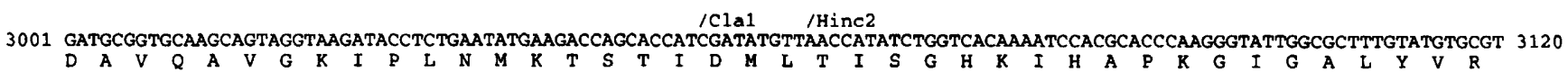

3121 CGCGGTGTGAGATTCCGTCCCTTGCTGATTGGTGGACACCAAGAACGTGGTCGCCGTGCAGGGACAGAGATGTACCCGGAATTGTCGCTTAGGTAAAGCCGCAGAGTTAGAATTAATT 3240

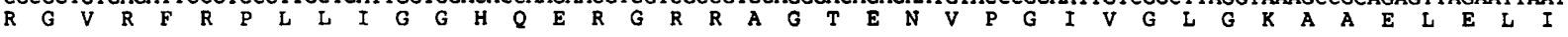

3241 CATATCGAAACAGCGATTAAGAAAGAAACAAGGCTGCGCGATCGCCTAGAACAAACCTTACTCGCTAAAATCCCTGACTGCGAAGTTAACGGTGACGTTACGCAGAGATTGCCCAACACC 3360

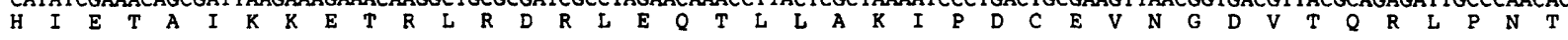

3361 ACCAACATCGGCTTCAAATACATTGAAGGCGAAGCGATTCTGCTCTCCTTAAACAAATATGGCATCTGTGCGTCGTCTGGTTCGGCTTGTACCTCTGGGTCGCTGGAACCATCCCACGTC 3480

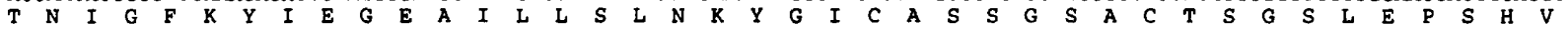

3481 CTCCGGGCAATGGGTTTACCATACACCACCCTCCACGGTTCGATTCGCTTCAGTCTTTGTCGCTACACTACAGAAGCTCAAATCGATCGCGTCATCGAAGTCATGCCCGAAATTGTCGAA 3600

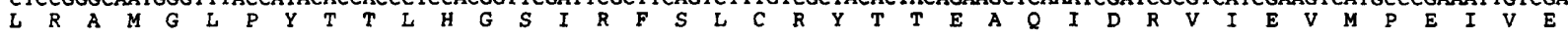

Fig. 2. For legend see facing page. 
3601 CGCCTCCGCGCCCTTTCCCCCTTCAAAAATGATGAAGCGGGTTGGTTGCAAGCCCAAGAACAAACATTGGCGCATCGTTAATTAGGGGCTGGGGACTAGGGACTGGGGACTGGGGACTAG 3720

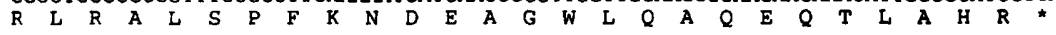

3721 GTAAGAGTTTTCCAGTACCCAATCCCCAATTCCCAATCCCACGAATCTAAAATCCAAAATCTAAAATCCAAAATAGGCCATGTGGGACTACACAGATAAAGTATTAGAACTGTTTTACG 3840 nitu> $M$ W $D$ Y $T$ D $K$ V L $E$ L $F$ Y $D$

3841 ATCCCAAGAATCAGGGAGTCATTGAAGATAACGGCGACCTGGCGTGAAGGTTGCCACCGGTGAAGTTGGGAGTATTGCTTGCGGTGATGCGCTGAGACTGCACATCAAGGTGAAGTAG 3960

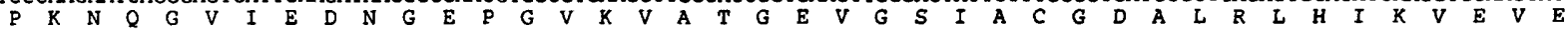

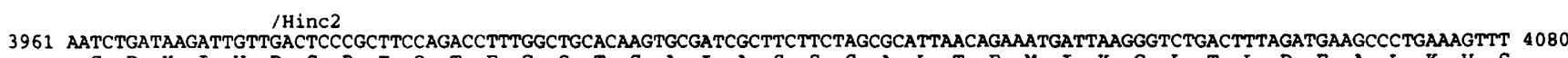

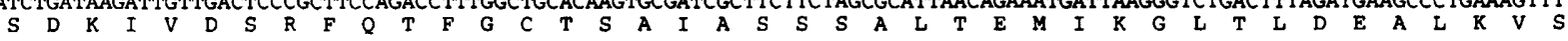
4081 CTAATAAAGACATTGCTGATTACCTCGGTGGCTTGCCAGAAGCCAAAATGCACTGCTCTGTGATGGGGCAAGAAGCTCTGGAAGCTGCTATCTATAACTATCGTGGCATTCCTCTCGCTA 4200

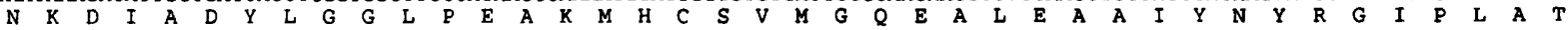
4201 CTCACGATGACGAAGATGAAGGTGCATTAGTTTGTACTTGCTTCGGTGGAGTGAAAATAAGGTACGTCGCATAGTTATCGAAAATGACCTTACTAGTGCGGAACAGGTAACAAATTACA 4320

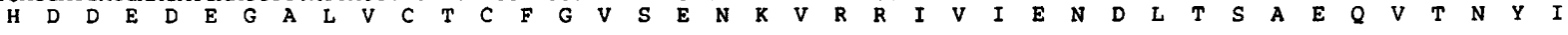

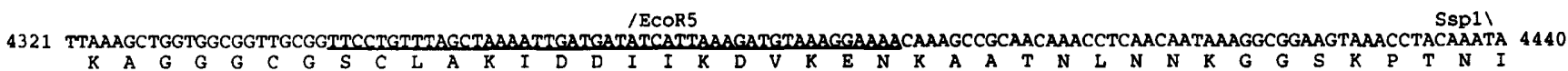
/ECOR5
4321
$\quad$ 441 TTCCTAATTCTGGCAAAAACGACCCCTCACCAACGTGCAGAAGATTGCCCTCATTCAAAAAGTATTAGACGAAGAAGTAAGACCCGTATTGATTGCCGACGGCGGAGATGTAGAACTCT 4560

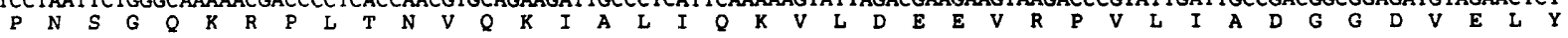

4561 ACGATGTAGACGGCGATATTGTCAAAGTAGTACTGCAAGGCGCGTGTGGCTCCTGTTCTAGTAGTACAGCCACCTTGAAAATAGCGATTGAATCCAGATTACGCGATCGCATTAATCCCA 4680

4561 ACGATGTAGACGGCGATATTGTCAAAGTAGTACTGCAAGGCGCGTGTGGCTCCTGTTCTAGTAGTACAGCCACCTTGAAAATAGCGATTGAATCCAGATTACGCGATCGCATTAATCCCA 4680

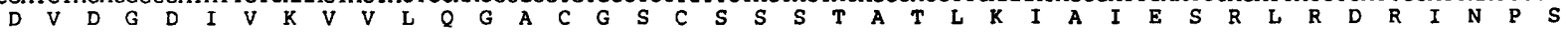
/Hinc2

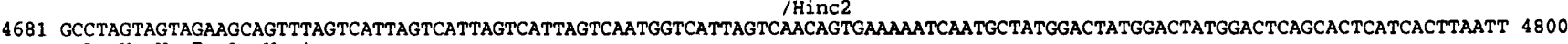
L V V E A V *

4801 ACATAACGAACCCATCATGAACAATAATTCTACCGGTTTTTCTGTAGAGCGATCGCCCCCTCTTCGGCGACGTTCTACAAACCCCTCACAGCCATAGCTCAACAGGCGTGAGATCCAAAC 4920 4921 ACAAAGACCGACCAACTAACCAACCAATTGCAGGAAAAGAGAACAATGACTGACGAAAACATTAGACAGATAGCTTTCTACGGTAAAGGCGGTATCGGTAAATCTACCACCTCCCAAAAC 5040

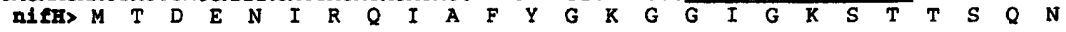

5041 ACCCTTGCAGCTATGGCAGAAATGGGTCAACGCATCATGATCGTAGGTTGCGACCCTAAAGCTGACTCCACCCGTTTGATGCTTCACGCTAAAGCGCAAACCACTGTACTTCACTTGGCT 5160

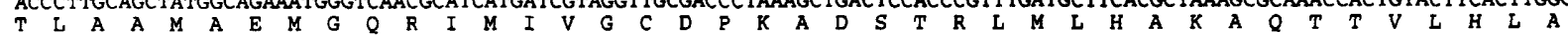

/Hinc2

5161 GCTGAACGCGGTGCAGTAGAAGACTTAGAACTACACGAAGTAATGTTGACCGGTTTCCGTGGCGTTCGTTGCGTAGAATCTGGTGGTCCAGAACCCGGTGTAGGTTGCGCCGGTCGTGGT 5280

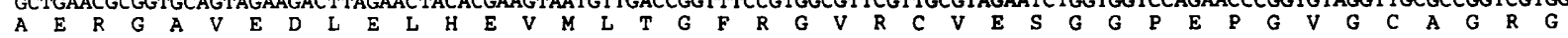

5281 ATCATCACCGCCATTAACTTCTTAGAAGAAAACGGTGCTTACCAAGACCTAGACTTCGTATCCTACGACGTATTGGGTGACGTTGTATGTGGTGGTTTCGCTATGCCTATCCGTGAAGGT 5400

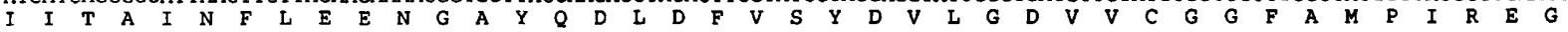

5401 AAAGCACAAGAAATCTACATCGTTACCTCTGGTGAAATGATGGCGATGTATGCTGCTAACAACATCGCTCGCGGTATTTTGAAATATGCTCACTCCGGTGGTGTACGCTTGGGGGTTTG 5520

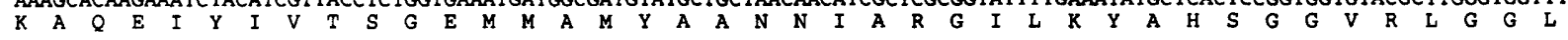

5521 ATTTGTAACAGCCGTAAAACTGACCGGGAAGCCGATTGATCGAAAACTTGGCTGAACGTTTGAACACTCAAATGATTCACTTCGTACCTCGTGACAACATCGTTCAACACGCTGAATTG 5640

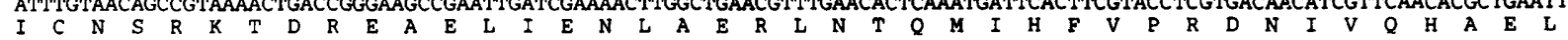

/Hinc2
5641 CGTCGGATGACTGTTACGAGTACGCACCAGACAGCAACCAAGGTCAAGAGTACCGTGCATTAGCCAAGAAAATCATCAACAACGACAAGCTCACCATTCCTACACCAATTGAAATGGAT 5760

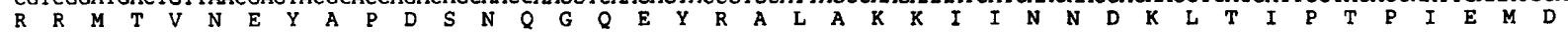

5761 GAACTCGAAGCCCTGTTGATCGAATACGGTATTCTTGATGATGATTCTAAGCACGCAGAAATCATCGGTAAGCCGCAGAAGCTACCAAATAGATAATGCCGTAATTKOCA\&AOAEGEAS 5880

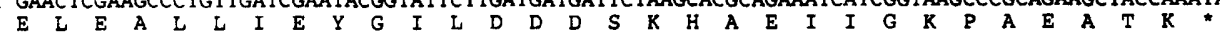

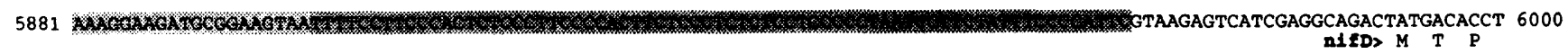

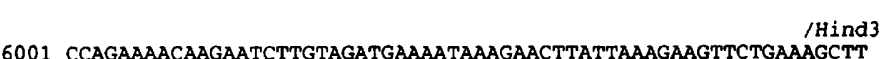

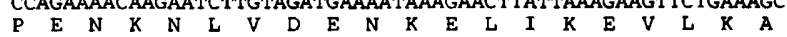

Fig. 2. The complete nucleotide sequence of a $6061 \mathrm{bp}$ region of $A$. azollae $1 \mathrm{a}$, including the nucleotide sequence and amino-acid translation of the nifB operon and the nifH gene. The following features are underlined in the nucleotide sequence: a putative IHF binding site (nucleotides 174-222); a stretch of 40 nucleotides that are present in $A$. azollae 1a but not in Anabaena 7120 (351-390); a sequence complementary to the nifB4 oligonucleotide (17-mer) used for primer extension analysis of the nifB promoter (426-442); a sequence identical to the Anabaena $712055 \mathrm{~kb}$ rearrangement breakpoint (2417-2421); a putative IHF binding site (nucleotides 4343-4390); and a sequence complementary to the nifH1 oligonucleotide (17-mer) used for primer extension analysis of the nifH promoter (5011-5027). The purine-rich (5868-5900) and pyrimidine-rich (5901-5971 regions are shaded. The locations of probable transcription start-points are indicated above the nucleotide sequence (O): at position 260 for the nifB operon, and at position 4836 for the nifH operon. The deduced amino-acid sequence of each gene product is given below the nucleotide sequence, with the identity of each indicated at the start. Restriction sites that were used for cloning and sequencing are shown above the nucleotide sequence. All of the Hincll sites in this region are shown.

of the sequence TAAT $\left({ }^{A} /{ }_{G}\right) G G$ (which we will name STRR4) are followed by eight copies of the sequence CAAT $(\mathrm{C} / \mathrm{T})$ AC (STRR5) (Fig. 3). Counterparts to the STRR4 sequences are not identifiable in Anabaena 7120 primarily because the extra DNA in the Anabaena 7120 genome interrupts the repeats (Fig. 3a). Anabaena 7120 has seven STRR1 sequences in the place of the eight STRR5 sequences (Fig. 3a). Both STRR4 and STRR5 
Table 1. Comparison of $A$. azollae 1a nif genes with those of the corresponding nif genes of Anabaena 7120

\begin{tabular}{|lcccc|}
\hline Gene & $\begin{array}{c}\text { Length } \\
\text { (bp) }\end{array}$ & $\begin{array}{c}\text { Nucleotide } \\
\text { identity (\%) }\end{array}$ & $\begin{array}{c}\text { Amino } \\
\text { acid } \\
\text { identity } \\
\mathbf{( \% )}\end{array}$ & $\begin{array}{c}\text { No. of } \\
\text { amino } \\
\text { acid } \\
\text { changes } \dagger\end{array}$ \\
\hline nifB & 1425 & $97 \cdot 4$ & $99 \cdot 6$ & $2(0)$ \\
fdxN & 348 & 93.4 & 93.1 & $7(5)$ \\
nifS & 1200 & 95.8 & $99 \cdot 0$ & $4(2)$ \\
nifU & 900 & 94.9 & 98.3 & $5(3)$ \\
nifH & 885 & 93.9 & 95.9 & $10(4)$ \\
\hline
\end{tabular}

* Pairwise identity with the corresponding gene product from Anabaena 7120.

†The number of conservative changes is indicated in parentheses.

sequences are similar to STRR1 sequences but can be distinguished from them. STRR4 sequences have three consecutive cytidine or guanine nucleotides but differ in the sequence which follows; STRR5 sequences lack three consecutive cytidine nucleotides.

The nifS-nif $U$ intergenic region is similar in length in both A. azollae $1 \mathrm{a}$ and Anabaena 7120. A. azollae 1a contains three sets of STRR sequences in this region (Fig. 3b). Five copies of the sequence $(\mathrm{A} / \mathrm{G})$ GGGACT are followed by five copies of $\mathrm{A}\left({ }^{\mathrm{A}} / \mathrm{G}_{\mathrm{G}}\right) \mathrm{T}\left({ }^{\mathrm{C}} / \mathrm{T}\right) \mathrm{CCC}$. Both of these are STRR1 sequences (Mazel et al., 1990), which could form a stem-loop structure with a free energy of $-36.6 \mathrm{kcal}$ $\mathrm{mol}^{-1}\left(-153.1 \mathrm{~kJ} \mathrm{~mol}^{-1}\right)$ in transcribed RNA. The third set of STRR sequences consists of four repeats of the sequence $\mathrm{C}(\mathrm{C} / \mathrm{T}) \mathrm{AAAAT}$ (STRR6). By contrast, Anabaena 7120 has six STRR1 sequences in the nifS-nifU intergenic region corresponding to the second set in $A$. azollae 1a. In Anabaena 7120 , the region corresponding to the first set of $A$. azollae 1a STRR1 sequences is best characterized as a purine-rich region with five repeats of the general sequence $R_{6} Y$, where $R$ stands for a purine and $\mathrm{Y}$ for a pyrimidine. This feature was not described previously (Mulligan \& Haselkorn, 1989). There are no counterparts to the STRR6 sequences in Anabaena 7120 (Fig. 3b).

In contrast to the above intergenic regions, the nifU-nifH region in $A$. azollae $1 \mathrm{a}$ is longer than that in Anabaena 7120 (Fig. 3c). It contains three sets of STRR sequences: seven copies of TAGTCA $\left({ }^{\mathrm{A}} / \mathrm{T}\right.$ ) (STRR3); three copies of ATGGACT (STRR7); and two copies of CA $\left({ }^{\mathrm{T}} / \mathrm{G}\right.$ ) CACT (STRR8). The nif U-nifH intergenic regions of $A$. azollae $1 \mathrm{a}$ and Nostoc 6720 are identical. Our analysis of the STRR structure of this region differs from that of Beesley et al. (1994). STRR sequences have not been described in the nif $U$-nifH intergenic region of Anabaena 7120 before, but four copies of the sequence CAGTTAA (STRR9) occur (Fig. 3c). Six copies of STRR9 occur in Anabaena L31 (Murphy et al., 1993). The STRR3 and STRR9 sequences are similar.

There are no tandemly repeating sequences in the intergenic region between nifH and nifD in either $A$. azollae 1a or Anabaena 7120, although a poorly conserved repeat was noted in Anabaena L31 (Murphy et al., 1993). Instead, this region is distinctive for the presence of a purine-rich tract followed immediately by a pyrimidinerich tract of nucleotides. In $A$. azollae 1a, a $33 \mathrm{bp} 85 \%$ purine-rich region is followed by a $71 \mathrm{bp} 90 \%$ pyrimidinerich region. In Anabaena 7120 , a 31 bp $85 \%$ purine-rich tract is followed by an $82 \%$ pyrimidine-rich tract. In Anabaena L31 a $32 \mathrm{bp} 81 \%$ purine-rich tract is followed by a $71 \mathrm{bp} 83 \%$ pyrimidine-rich region (Murphy et al., 1993). Nostoc 6720 has similar regions (Beesley et al., 1994).

\section{Other sequences}

In Anabaena 7120 , the $f d x N$ gene in the vegetative cell chromosome is interrupted by a $55 \mathrm{~kb}$ excisable element (Golden et al., 1987, 1988; Mulligan et al., 1988; Mulligan \& Haselkorn, 1989). The $f d x N$ gene of $A$. azollae $1 \mathrm{a}$ is intact in vegetative cell DNA and it contains a sequence that is identical to one of the Anabaena 7120 rearrangement breakpoints (Golden et al., 1987). The nucleotide sequence of the $5 \mathrm{bp}$ core sequence as well as $18 \mathrm{bp}$ on each side are completely conserved. All of the conserved bases that

Table 2. Pairwise comparisons of nifH gene sequences to nifH of $A$. azollae 1a

\begin{tabular}{|lcccl|}
\hline Organism & $\begin{array}{c}\text { Nucleotide } \\
\text { identity (\%)* }\end{array}$ & $\begin{array}{c}\text { Amino acid } \\
\text { identity (\%)* }\end{array}$ & $\begin{array}{c}\text { No. of amino } \\
\text { acid } \\
\text { changes } \dagger\end{array}$ & Reference \\
\hline Anabaena 7120 & 93.9 & 95.9 & $10(4)$ & Mevarech et al. $(1980)$ \\
Anabaena L31 & $94 \cdot 0$ & $98 \cdot 3$ & $4(2)$ & Murphy et al. (1993) \\
Nostoc 6720 & $99 \cdot 7$ & $99 \cdot 7$ & $1(0)$ & Beesley et al. (1994) \\
$\begin{array}{l}\text { Plectonema } \\
\text { boryanum }\end{array}$ & $77 \cdot 7$ & 84.5 & $41(13)$ & Fujita et al. $(1991)$ \\
\hline
\end{tabular}

* Pairwise identity with the nif $H$ gene or gene product of $A$. azollae 1 a.

$\dagger$ The number of conservative changes is indicated in parentheses. 


\section{(a) $\operatorname{nifB}-f d x N$}

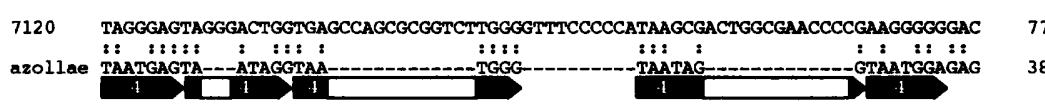

7120 TAGGCAAATTACCCAATCCCCAATCCCCGATACCTAATCCCCAATCTCCAATCC-CCAATCAACAAGGAGAATAATC

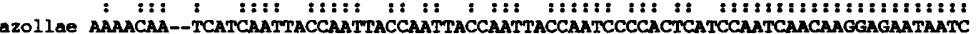
azollae AAAACAA--TCATCAATTACCAATPACCAATTACCAATTACCAATCCCCACTCATCCAATCAACAMGGAGAATAMTC
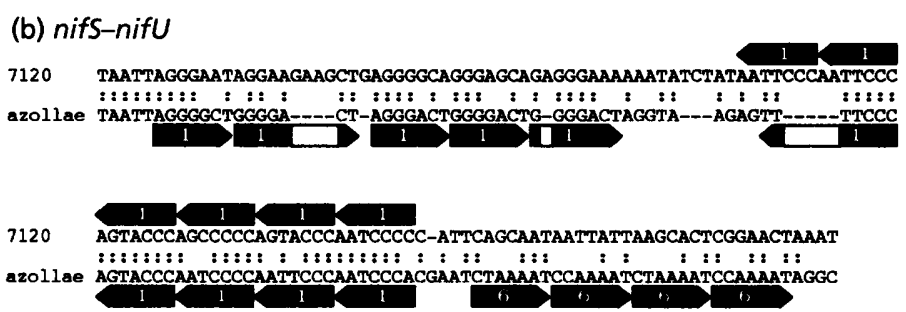

(c) nifU-nifH

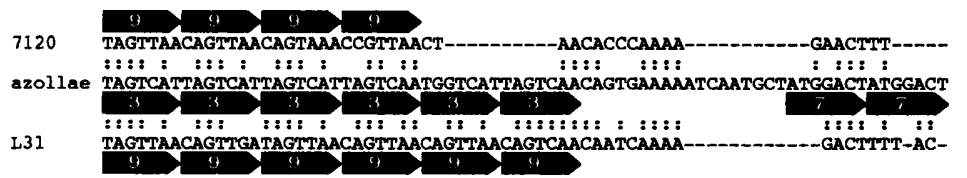

7120

azollae $\mathbf{1 3 1}$
TGGTTTPTCTGTG AATTACATAACGAACCCATCATGAACAATAATTCTACCGGTTTTTCTGTA

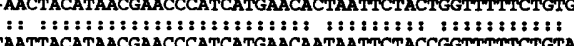

Fig. 3. Alignment of the nucleotide sequence of the intergenic regions. Each of the intergenic regions from $A$. azollae 1a (azollae) was aligned with the corresponding region from Anabaena $\mathbf{7 1 2 0}$ (7120) or Anabaena L31 (L31) using the ALIGN program of the FASTA program package. Dashes indicate gaps that were introduced during alignment. STRR sequences are indicated by arrows above or below the nucleotide sequence; different STRR sequences are denoted by the number within each arrow. The sequences are numbered beginning with the start of the sequence that is listed. (a) Alignment of the complete nucleotide sequence of the nifB$f d x N$ intergenic region (nucleotides 2013-2125 in Fig. 2). (b) Alignment of the complete nucleotide sequence of the nifsnifU intergenic region (3679-3800). (c) Alignment of a partial nucleotide sequence of the nifU-nifH intergenic region (4701-4846). The location of the probable nifH transcription start-point is also indicated (O). surround the central 5 bp core in Anabaena 7120 (Golden et al., 1987) are conserved in the $A$. azollae 1a sequence.

We searched the $A$. azollae 1 a nucleotide sequence for the presence of integration host factor (IHF) binding sites. Two moderately high scoring sites were found. One is located near the nifB promoter and is centred approximately $65 \mathrm{bp}$ upstream of the putative nifB transciption startpoint (Fig. 2). This site has a similarity score of 56.5 . A second site, which is in the opposite orientation, located near the end of the nif $U$ gene (Fig. 2) is centred $470 \mathrm{bp}$ upstream of the nifH promoter. This site has a similarity score of 53.8 . The similarity scores of known IHF sites range from 46 to 77 (Goodrich et al., 1990) with, for example, the bacteriophage lambda att $P$ H2 site having a similarity score of $56 \cdot 3$.

\section{Expression in A. azollae 1a}

Expression of the nifB and nif $H$ operons in $A$. azollae $1 \mathrm{a}$ was examined first by blot hybridization analysis. A nifHspecific RNA probe was used to probe membrane blots of RNA isolated from non-induced (N-replete) and induced (N-depleted) cultures of $A$. azollae 1a. nifH-specific RNA was not present in non-induced cultures but at least two specific transcripts were detectable from induced cultures (Fig. 4a). The estimated sizes of these transcripts $(1.2 \mathrm{~kb}$ and $2.6 \mathrm{~kb}$ ) could correspond to RNA species that contain nif $H$ and nif $H D$, respectively. A larger transcript that could correspond to a nifHDK transcript was observed on some other blots (not shown). Similar patterns and sizes of transcript have been reported for nif $H$ transcription in Anabaena 7120 (Haselkorn et al., 1983, 1986). We were unable to detect nifB-specific transcripts by blot hybridization analysis.

The startpoint of transcription for both the nifB and nifH operons was determined by primer extension analysis. When a nifH-specific oligonucleotide primer was extended, three major products that differed in size by only a few bases were observed (Fig. 4b). All three products map within a region of five consecutive thymine nucleotides. Since this region potentially forms an unstable RNA:DNA hybrid, it is likely that the true startpoint is elsewhere, perhaps the guanine nucleotide at position 4836 (Fig. 2). Similar results were obtained previously for $\mathrm{S} 1$ nuclease mapping of the Anabaena 7120 nifH transcription startpoint (Haselkorn et al., 1983).

The results of primer extension mapping of the nifB transcription startpoint were not as clear cut. When a nifBspecific oligonucleotide primer was used, extension products were observed using RNA isolated from both uninduced and induced filaments (Fig. 4c). One of the products, however, was more abundant when RNA from induced filaments was used. If this product is due to a 
(a)

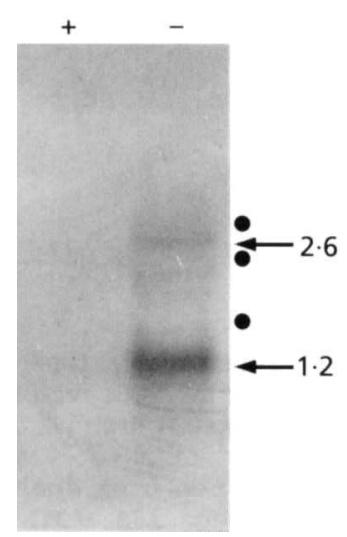

(b)

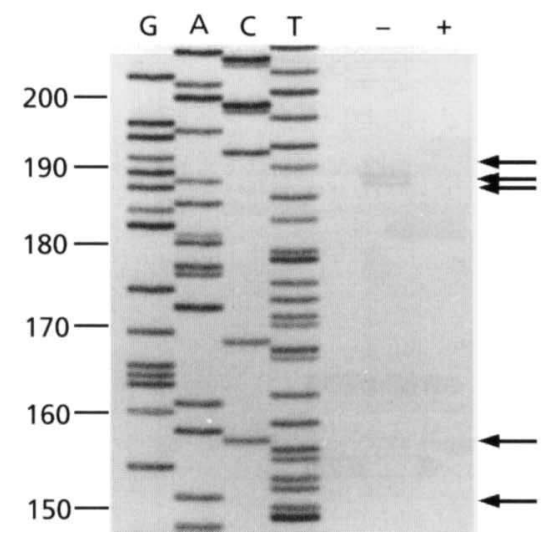

(c)

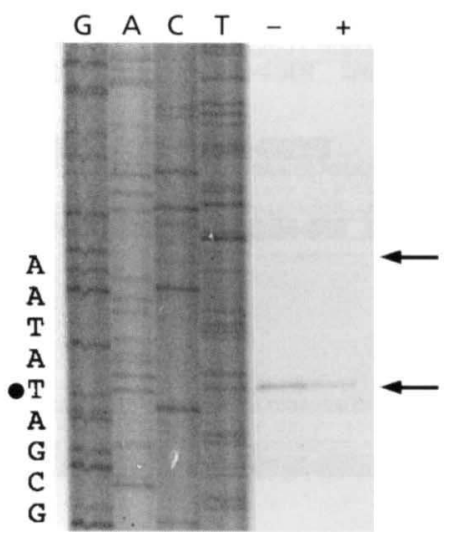

Fig. 4. Transcription of the nifH and nifB operons. RNA isolated from cultures of $A$. azollae 1a grown under nitrogen replete $(+)$ or nitrogen starved $(-)$ conditions was used for RNA:RNA hybridization analysis and primer extension experiments. (a) RNA:RNA analysis of RNA probed with a nifH-specific RNA probe. The estimated sizes (in kb) of the two major hybridizing bands are shown. The locations of rRNA bands are shown by the dots. (b) Primer extension analysis using the nifH1 primer (Fig. 2). Arrows indicate the positions of extended products. A DNA sequencing ladder was used to calibrate the length of the extended products; sizes of the sequencing bands are indicated. (c) Primer extension analysis using the nifB4 primer (Fig. 2). Arrows indicate the positions of extended products. A DNA sequencing ladder was generated using the same primer; the sequence of the region surrounding the major extended product is shown and the putative startpoint is indicated by a dot.

genuine induced transcript, then the startpoint mapped by it is the same as that previously observed in Anabaena 7120 (Mulligan \& Haselkorn, 1989).

\section{DISCUSSION}

\section{STRR sequences in cyanobacteria}

A major feature of interest in cyanobacterial genomes may well be the variety of STRR sequences that are found mostly in the non-coding regions rather than any variation of the coding regions. The function of the STRR sequences is not known. It has been suggested that they may regulate transcription termination (Haselkorn \& Buikema, 1992) or be the target of DNA-binding proteins responsible for chromosome maintenance in the cell (Mazel et al., 1990). Alternatively, it is possible that these sequences serve as binding sites for proteins involved in mRNA stability or processing.

The prevalence and conservation of STRR sequences in heterocyst-forming cyanobacteria suggests that these sequences have some important function. And yet, the number and sequence of STRR sequences at comparable locations in closely related strains, such as the nifU-nifH intergenic region of Anabaena $7120, A$. azollae $1 \mathrm{a}$ and Anabaena L31, are quite different. It is unlikely that these sequences play a direct role in the regulation of gene expression. Otherwise, it would be necessary to propose that different regulatory mechanisms exist in each strain of heterocyst-forming cyanobacteria depending on its exact complement of STRR sequences. In any case, the high conservation of STRR sequences suggests that they may be valuable taxonomic tools for the study of heterocystforming cyanobacteria. It may even be more informative to analyse and compare the intergenic regions (using, for example, PCR techniques) than the coding regions.

One clue to the function and/or the origin of STRR sequences may come from Nostoc commune and related cyanobacteria. $N$. commune UTEX 584 is notable for the presence of a cyanoglobin gene between nif $U$ and nif $H$ (Potts et al., 1992). A second cyanobacterial strain, Nostoc 8820 , that contains a $g l b N$ gene has been sequenced in our laboratory (T. J. Belbin \& M. E. Mulligan, unpublished results). There are no STRR sequences in either the $n i f U-g l b N$ or the $g l b N-n i f H$ intergenic regions of Nostoc 8820. The presence of STRR sequences in many cyanobacteria (at this location, at least) might have arisen as a result of the loss of $g l b N$ from an ancestral strain or they may have been lost as a result of the gain of $g l b N$. In either of these cases, STRR sequences would reflect unexpected genome plasticity. A similar observation was made in the case of the variation in STRR sequences found in the nifJ gene (Bauer et al., 1993).

\section{nif gene expression in cyanobacteria}

Our motivation for commencing this work was the desire to collect nucleotide sequence data for nif promoters in different heterocyst-forming cyanobacteria. It is not possible to define a nif promoter consensus sequence from the results reported in this paper with any more certainty than has been reported previously (Borthakur et al., 1990; Mulligan \& Haselkorn, 1989). However, it is possible to delimit the extent of nucleotide sequence that must contain the core nifH promoter. Fig. 3(c) shows that the alignment of nucleotide sequence in the vicinity of the nifH promoter extends only $38 \mathrm{bp}$ upstream of the transcription startpoint before the presence of STRR 
sequences in the $A$. azollae 1a genome precludes all further alignment. All sequence elements that are required to define the nifH promoter should occur within these $38 \mathrm{bp}$. Although there are no STRR sequences upstream of the nifB promoter that can be used in the same way, it is interesting that the distance between the nif $B$ transcription startpoint and the adjacent putative IHF binding site is also $40 \mathrm{bp}$.

If the IHF binding sites near the nifB and nifH promoters are functional sites in cyanobacteria, they may have interesting implications for cyanobacterial nif transcription. IHF is required for nif-specific transcription in many purple bacteria, where it functions to bend DNA, permitting close contact between an activator protein (e.g. NifA) and RNA polymerase containing the $\sigma^{54}$ subunit (Weiss et al., 1992). The biochemical components required for nif-specific transcription in cyanobacteria, however, have not yet been characterized.

\section{Identity of A. azollae 1a}

The suspicion that isolates of cyanobacteria cultured from the Azolla symbiosis might not represent the true symbiotic cyanobacterium first arose when the nif-specific Southern hybridization patterns of freshly isolated symbionts from four geographically diverse Euazolla species were examined (Franche \& Cohen-Bazire, 1985). All four were closely related to one another but were not related to a cultured isolate from Azolla filiculoides. Every study of cultured isolates has since obtained the same result: cultured isolates do not resemble the symbiont (Franche \& Cohen-Bazire, 1987; Gebhardt \& NierzwickiBauer, 1991; Meeks et al., 1988). Gebhardt \& NierzwickiBauer (1991) posed the question: what is actually being cultured? On the basis of morphology, lectin binding and the characterization of allozymes, Zimmerman et al. (1989) concluded that five independent cultured isolates were identical to each other and to Anabaena 29413. A similar conclusion was reached from RFLP analysis of three independent isolates (Gebhardt \& Nierzwicki-Bauer, 1991). Restriction digests of $A$. azollae 1a and Anabaena 29413 DNA are indistinguishable (not shown) and Southern blots of these digests always show identical patterns. We have observed this using both nif $H$ and nif $B$ probes (not shown). Others have observed the same result, for example with a bet $\mathrm{R}$ probe (Buikema \& Haselkorn, 1991), with a nifJ probe (Bauer et al., 1993), and with an STRR probe (Bauer et al., 1993). Finally, the nucleotide sequence of the $A$. azollae 1 a nif genes is more than $99 \%$ identical with that of Anabaena 29413 (Lyons \& Thiel, 1995).

It is not clear, however, where the cultured isolates come from. Are they minor symbionts or are they surface contaminants? It has been argued that it is unlikely that the cultured isolates are due to contaminating epiphytic organisms associated with the surface of the ferns precisely because the same strain can be cultured from geographically distinct Azolla spp. (Gebhardt \& Nierzwicki-Bauer, 1991). However, a tenacious Anabaena epiphyte, which resisted surface sterilization procedures, was frequently found on the surfaces of root nodules from the cycad Zamia integrifolia (Zimmerman \& Rosen, 1992), a different example of a cyanobacterial symbiosis. The very high sequence similarity between the naturally free-living species Anabaena 29413 and cultured isolates such as Anabaena azollae 1a suggests that further study is needed to resolve the issue.

\section{NOTE ADDED IN PROOF}

The cyanobacterium studied by Beesley $e t$ al. (1994) as Nostoc sp. PCC 6720 should be considered to be Anabaena variabilis PCC 7937 (R. J. Smith, personal communication). All references to and comparisons with Nostoc 6720 in the paper should therefore be interpreted as being with Anabaena variabilis PCC 7937.

\section{ACKNOWLEDGEMENTS}

We thank Teresa Thiel (University of Missouri at St Louis) for communicating results prior to publication and Bill Buikema (University of Chicago) and Jeff Elhai (Florida International University) for comments on the manuscript. The work reported in this paper was funded in part by a grant from the Medical Research Council of Canada and from Memorial University of Newfoundland.

\section{REFERENCES}

Ausubel, F. M., Brent, R., Kingston, R. E., Moore, D. D., Seidman, J. G., Smith, J. A. \& Struhl, K. (1987). Current Protocols in Molecular Biology. New York: Greene Publishing Associates \& WileyInterscience.

Bauer, C. C., Scappino, L. \& Haselkorn, R. (1993). Growth of the cyanobacterium Anabaena on molecular nitrogen: NifJ is required when iron is limited. Proc Natl Acad Sci US A 90, 8812-8816.

Beesley, C. E., Smith, R. J., Temple, S. J. \& Lea, P. J. (1994). Cloning and nucleotide sequence of the gene encoding dinitrogenase reductase (nifH) from the cyanobacterium Nostoc 6720. Biocbim Biophys Acta 1219, 548-550.

Belknap, W. R. \& Haselkorn, R. (1987). Cloning and light regulation of expression of the phycocyanin operon of the cyanobacterium Anabaena. EMBO J 6, 871-884.

Ben-Porath, J. \& Zehr, J. P. (1994). Detection and characterization of cyanobacterial nifH genes. Appl Environ Microbiol 60, 880-887.

Ben-Porath, J., Carpenter, E. J. \& Zehr, J. P. (1993). Genotypic relationships in Trichodesmium (Cyanophyceae) based on nifH sequence comparisons. $J$ Phycol 29, 806-810.

Borthakur, D., Basche, M., Buikema, W. J., Borthakur, P. B. \& Haselkorn, R. (1990). Expression, nucleotide sequence and mutational analysis of two open reading frames in the nif gene region of Anabaena sp. strain PCC 7120. Mol \& Gen Genet 221, 227-234.

Buikema, W. J. \& Haselkorn, R. (1991). Characterization of a gene controlling heterocyst differentiation in the cyanobacterium Anabaena 7120. Genes \& Dev 5, 321-330.

Defrancesco, N. \& Potts, M. (1988). Cloning of nifHD from Nostoc commune UTEX 584 and of a flanking region homologous to part of the Arotobacter vinelandii nifU gene. J Bacteriol 170, 3297-3300.

Evans, H. J. \& Burris, R. H. (1992). Highlights in biological nitrogen fixation. In Biological Nitrogen Fixation, pp. 1-42. Edited by G. Stacey, R. H. Burris \& H. J. Evans. New York: Chapman \& Hall. 
Franche, C. \& Cohen-Bazire, G. (1985). The structural nif genes of four symbiotic Anabaena azollae show a highly conserved physical arrangement. Plant Sci 39, 125-131.

Franche, C. \& Cohen-Bazire, G. (1987). Evolutionary divergence in the $n i f \mathrm{H}, \mathrm{D}, \mathrm{K}$ gene region among nine symbiotic Anabaena azollae and between Anabaena azollae and some free-living heterocystous cyanobacteria. Symbiosis 3, 159-178.

Fujita, Y., Takahashi, Y., Shonai, F., Ogura, Y. \& Matsubara, H. (1991). Cloning, nucleotide sequences and differential expression of the nifH and nifH-like $(f r \times C)$ genes from the filamentous nitrogenfixing cyanobacterium Plectonema boryanum. Plant Cell Pbysiol 32, 1093-1106.

Gebhardt, J. S. \& Nierzwicki-Bauer, S. A. (1991). Identification of a common cyanobacterial symbiont associated with Azolla spp. through molecular and morphological characterization of freeliving and symbiotic cyanobacteria. Appl Environ Microbiol 57, 2141-2146.

Gilbert, D. (1993). The global library. Trends Biocbem Sci 18, 107-108.

Golden, J. W., Mulligan, M. E. \& Haselkorn, R. (1987). Different recombination site specificity of two developmentally regulated genome rearrangements. Nature 327, 526-529.

Golden, J. W., Carrasco, C. D., Mulligan, M. E., Schneider, G. J. \& Haselkorn, R. (1988). Deletion of a 55-kilobase-pair DNA element from the chromosome during heterocyst differentiation of Anabaena sp. strain PCC 7120. J Bacteriol 170, 5034-5041.

Goodrich, J. A., Schwartz, M. L. \& McClure, W. R. (1990). Searching for and predicting the activity of sites for DNA binding proteins: compilation and analysis of the binding sites for Escherichia coli Integration Host Factor (IHF). Nucleic Acids Res 18, 4993-5000.

Haselkorn, R. \& Buikema, W. J. (1992). Nitrogen fixation in cyanobacteria. In Biological Nitrogen Fixation, pp. 166-190. Edited by G. Stacey, R. H. Burris \& H. J. Evans. New York: Chapman \& Hall.

Haselkorn, R., Rice, D., Curtis, S. E. \& Robinson, S. J. (1983). Organization and transcription of genes important in Anabaena heterocyst differentiation. Ann Microbiol (Paris) 134B, 181-193.

Haselkorn, R., Golden, J. W., Lammers, P. J. \& Mulligan, M. E. (1986). Developmental rearrangement of cyanobacterial nitrogenfixation genes. Trends Genet 2, 255-259.

Hirschberg, R., Samson, S. M., Kimmel, B. E., Page, K. A., Collins, J. J., Myers, J. A. \& Yarbrough, L. R. (1985). Cloning and characterization of nitrogenase genes from Anabaena variabilis. J Biotechnol 2, 23-37.

Jaeger, J. A., Turner, D. H. \& Zuker, M. (1989). Predicting optimal and suboptimal secondary structure for RNA. Metbods Enzymol 183, 281-306.

Jobling, M. G. \& Holmes, R. K. (1990). Construction of vectors with the $\mathrm{p} 15 \mathrm{a}$ replicon, kanamycin resistance, inducible $\mathrm{lac} Z a$ and pUC18 or pUC19 multiple cloning sites. Nucleic Acids Res 18, $5315-5316$.

Luftig, R. \& Haselkorn, R. (1967). Morphology of a virus of bluegreen algae and properties of its deoxyribonucleic acid. $J$ Virol 1 , 344-361.

Lyons, E. M. \& Thiel, T. (1995). Characterization of nifB, nifS, and nif $U$ genes in the cyanobacterium Anabaena variabilis: $\mathrm{NifB}$ is required for the vanadium-dependent nitrogenase. $J$ Bacteriol 177, 1570-1575.

Mathur, M. \& Tuli, R. (1990). Cluster analysis of genes for nitrogen fixation from several diazotrophs. J Genet 69, 67-78.
Mazel, D., Houmard, J., Castets, A. M. \& Tandeau de Marsac, N. (1990). Highly repetitive DNA sequences in cyanobacterial genomes. J Bacteriol 172, 2755-2761.

Meeks, J. C., Joseph, C. M. \& Haselkorn, R. (1988). Organization of the nif genes in cyanobacteria in symbiotic association with Azolla and Anthoceros. Arch Microbiol 150, 61-71.

Mevarech, M., Rice, D. \& Haselkorn, R. (1980). Nucleotide sequence of a cyanobacterial nif $H$ gene coding for nitrogenase reductase. Proc Natl Acad Sci US A 77, 6476-6480.

Mulligan, M. E. \& Haselkorn, R. (1989). Nitrogen-fixation (nif) genes of the cyanobacterium Anabaena sp. strain PCC 7120: the nifB-fdxN-nifS-nifU operon. J Biol Chem 264, 19200-19207.

Mulligan, M. E., Buikema, W. J. \& Haselkorn, R. (1988). Bacterialtype ferredoxin genes in the nitrogen fixation regions of the cyanobacterium Anabaena sp. strain PCC 7120 and Rbizobium meliloti. J Bacteriol 170, 4406-4410.

Murphy, S. T., Jackman, D. M. \& Mulligan, M. E. (1993). Cloning and nucleotide sequence of the gene for dinitrogenase reductase (nifH) from the heterocyst-forming cyanobacterium Anabaena sp. L31. Biochim Biophys Acta 1171, 337-340.

Newton, J. W. \& Herman, A. I. (1979). Isolation of cyanobacteria from the aquatic fern, Azolla. Arch Microbiol 120, 161-165.

Parker, M. (1993). Biological data access through Gopher. Trends Biocbem Sci 18, 485-486.

Pearson, W. R. \& Lipman, D. J. (1988). Improved tools for biological sequence analysis. Proc Natl Acad Sci USA 85, 2444-2448.

Peters, G. A. \& Meeks, J. C. (1989). The Azolla-Anabaena symbiosis: basic biology. Annu Rev Plant Pbysiol Plant Mol Biol 40, 193-210.

Potts, M., Angeloni, S. V., Ebel, R. E. \& Bassam, D. (1992). Myoglobin in a cyanobacterium. Science 256, 1690-1692.

Reed, K. C. \& Mann, D. A. (1985). Rapid transfer of DNA from agarose gels to nylon membranes. Nucleic Acids Res 13, 7207-7221.

Rice, D., Mazur, B. J. \& Haselkorn, R. (1982). Isolation and physical mapping of nitrogen fixation genes from the cyanobacterium Anabaena 7120. J Biol Chem 257, 13157-13163.

Sambrook, J., Fritsch, E. F. \& Maniatis, T. (1989). Molecular Cloning: a Laboratory Manual. Cold Spring Harbor, NY: Cold Spring Harbor Laboratory.

Thiel, T. (1993). Characterization of genes for an alternative nitrogenase in the cyanobacterium Anabaena variabilis. $J$ Bacteriol 175, 6276-6286.

Weiss, D. S., Klose, K. E., Hoover, T. R., North, A. K., Porter, S. C., Wedel, A. B. \& Kustu, S. (1992). Prokaryotic transcriptional enhancers. In Transcriptional Regulation, pp. 667-694. Edited by S. L. McKnight \& K. R. Yamamoto. Cold Spring Harbor, NY: Cold Spring Harbor Laboratory.

Zimmerman, W. J. \& Rosen, B. H. (1992). Cyanobiont diversity within and among cycads of one field site. Can J Microbiol 38, $1324-1328$

Zimmerman, W. J., Rosen, B. H. \& Lumpkin, T. A. (1989). Enzymatic, lectin, and morphological characterization and classification of presumptive cyanobionts from Azolla Lam. New Phytol 113, 497-503.

Zuker, M. (1989). On finding all suboptimal foldings of an RNA molecule. Science 244, 48-52.

Received 24 January 1995; revised 24 April 1995; accepted 3 May 1995. 